\title{
Kinks in a non-linear massive sigma model
}

\author{
A. Alonso-Izquierdo ${ }^{a}$, M. A. Gonzalez Leon ${ }^{a}$ and J. Mateos Guilarte ${ }^{b}$ \\ ${ }^{a}$ Departamento de Matematica Aplicada and IUFFyM, Universidad de Salamanca, SPAIN \\ ${ }^{b}$ Departamento de Fisica and IUFFyM, Universidad de Salamanca, SPAIN
}

\begin{abstract}
We describe the kink solitary waves of a massive non-linear sigma model with an $\mathbb{S}^{2}$ sphere as the target manifold. Our solutions form a moduli space of non-relativistic solitary waves in the long wavelength limit of ferromagnetic linear spin chains.
\end{abstract}

PACS numbers: 11.10. Lm, 11.27. +d

\section{THE MASSIVE NON-LINEAR $\mathbb{S}^{2}$-SIGMA MODEL}

In this letter we shall concentrate on the 1D nonlinear $\mathbb{S}^{2}$-sigma model: (a) The space-time is the $(1+1)$ dimensional $\mathbb{R}^{1,1}$ Minkowski space. $x^{\mu}, \mu=0,1\left(x^{0} \equiv\right.$ $\left.t, x^{1} \equiv x\right)$ denotes a point in $\mathbb{R}^{1,1}$. We choose the metric in the form $g^{\mu \nu}=\operatorname{diag}(1,-1)$ and the d'Alembertian reads: $\square=\frac{\partial^{2}}{\partial t^{2}}-\frac{\partial^{2}}{\partial x^{2}}$. (b) The target (internal) space is the $\mathbb{S}^{2}$-sphere. This is in contrast with the original Gell-Mann/Lèvy model where the target space is $\mathbb{S}^{3}[1]$. Three scalar fields $\Phi=\left(\phi_{a}\right), a=1,2,3$, define a map $\Phi: \mathbb{R}^{1,1} \rightarrow \mathbb{S}^{2}$ if they are constrained to the surface in $\mathbb{R}^{3}$ :

$$
\phi_{1}^{2}\left(x^{\mu}\right)+\phi_{2}^{2}\left(x^{\mu}\right)+\phi_{3}^{2}\left(x^{\mu}\right)=R^{2}
$$

The action

$$
S=\int d t d x\left\{\frac{1}{2} g^{\mu \nu} \sum_{a=1}^{3} \frac{\partial \phi_{a}}{\partial x^{\mu}} \frac{\partial \phi_{a}}{\partial x^{\nu}}\right\}
$$

seems to be simple but together with the constraint (11) governs the complicated non-linear dynamics of two Goldstone bosons with coupling constant $\frac{1}{R}$. In one spatial dimension, however, Goldstone particles do not exist [2]. The infrared asymptotics of the (11)-(2) system induces a potential energy density that we choose as:

$$
V\left(\phi_{1}, \phi_{2}\right)=\frac{\lambda^{2}}{2} \phi_{1}^{2}(t, x)+\frac{\gamma^{2}}{2} \phi_{2}^{2}(t, x)
$$

giving masses $\lambda$ and $\gamma$ to the massless excitations.

With no loss of generality, we assume that: $\lambda^{2} \geq \gamma^{2}>$ 0 , and we define the non-dimensional parameter: $\sigma^{2}=$ $\frac{\gamma^{2}}{\lambda^{2}}, 0<\sigma^{2} \leq 1$, measuring the ratio between particle masses. We also re-scale the space-time coordinates to address non-dimensional variables: $x^{\mu} \rightarrow \frac{x^{\mu}}{\lambda}$. Solving the constraint the action reads

$$
\begin{aligned}
S & =\frac{1}{2} \int d t d x\left\{\sum_{\alpha=1}^{2} \frac{\partial \phi_{\alpha}}{\partial x^{\mu}} \frac{\partial \phi_{\alpha}}{\partial x_{\mu}}-\phi_{1}^{2}-\sigma^{2} \phi_{2}^{2}\right. \\
& \left.+\frac{\sum_{\alpha=1}^{2}\left(\phi_{\alpha} \partial^{\mu} \phi_{\alpha}\right) \sum_{\beta=1}^{2}\left(\phi_{\beta} \partial_{\mu} \phi_{\beta}\right)}{R^{2}-\phi_{1}^{2}-\phi_{2}^{2}}\right\}
\end{aligned}
$$

Despite the potential energy density being quadratic, there are two homogeneous minima of the action (vacua): the North and South Poles: $\Phi^{A}=(0,0, R), \Phi^{\bar{A}}=$ $(0,0,-R)$. Thus, the discrete symmetry of the action(4) $\mathbb{Z}_{2} \times \mathbb{Z}_{2} \times \mathbb{Z}_{2}$ generated by $\phi_{a} \rightarrow-\phi_{a}, a=1,2,3$ is spontaneously broken to $\mathbb{Z}_{2} \times \mathbb{Z}_{2}$ (generated by $\phi_{\alpha} \rightarrow-\phi_{\alpha}$, $\alpha=1,2$ ). If the two masses were equal, this unbroken symmetry would become the $S O(2)$ rotation group around the North-South Pole axis.

\section{TOPOLOGICAL KINKS}

Using spherical coordinates, $\phi_{1}=R \cos \varphi \sin \theta, \phi_{2}=$ $R \sin \varphi \sin \theta, \phi_{3}=R \cos \theta$, in the chart $\mathbb{S}^{2}-\{\bar{A}\}$ of $\mathbb{S}^{2}$ the energy of static configurations: $\theta(t, x)=\theta(x) \in[0, \pi)$, $\varphi(t, x)=\varphi(x) \in[0,2 \pi), E=\int d x \mathcal{E}(\theta(x), \varphi(x))$, and the potential energy density read:

$$
\begin{aligned}
& E=\int d x\left\{\frac{R^{2}}{2}\left[\left(\frac{d \theta}{d x}\right)^{2}+\sin ^{2} \theta\left(\frac{d \varphi}{d x}\right)^{2}\right]+V(\theta, \varphi)\right\} \\
& V(\theta(x), \varphi(x))=\frac{R^{2}}{2} \sin ^{2} \theta(x)\left(\sigma^{2}+\left(1-\sigma^{2}\right) \cos ^{2} \varphi(x)\right)
\end{aligned}
$$

The configuration space of the system $\mathcal{C}=$ $\left\{\operatorname{Maps}\left(\mathbb{R}, \mathbb{S}^{2}\right) / E<+\infty\right\}$ is formed by four disconnected sectors according to the tendency of every finite energy configuration towards either the North or South Pole at the extremes of the spatial line $x= \pm \infty$.

Solutions for which the temporal dependence is of the form

$$
\theta(t, x)=\theta\left(\frac{x-v t}{\sqrt{1-v^{2}}}\right), \varphi(t, x)=\varphi\left(\frac{x-v t}{\sqrt{1-v^{2}}}\right)
$$

for some velocity $v$, are solitary or traveling waves. Lorentz invariance provides all the solitary waves from the static solutions of the field equations

$$
\begin{aligned}
\frac{d^{2} \theta}{d x^{2}}-\frac{\sin 2 \theta}{2}\left(\frac{d \varphi}{d x}\right)^{2} & =\frac{\sin 2 \theta}{2}\left(\cos ^{2} \varphi+\sigma^{2} \sin ^{2} \varphi\right)(6) \\
\frac{d}{d x}\left(\sin ^{2} \theta \frac{d \varphi}{d x}\right) & =\frac{1-\sigma^{2}}{2} \sin ^{2} \theta \sin 2 \varphi
\end{aligned}
$$

On the orbits $\varphi_{K_{1}}(x)= \pm \frac{\pi}{2}$ (half meridians) the system (6)-(7) becomes the ODE of the pendulum and the 
separatrix trajectories between bounded and unbounded motion;

$$
-\frac{d^{2} \theta}{d x^{2}}+\frac{\sigma^{2}}{2} \sin 2 \theta=0 \Rightarrow \theta_{K_{1}}(x)=2 \arctan e^{ \pm \sigma\left(x-x_{0}\right)}
$$

are the solitary waves or kinks (at their center of mass) of finite energy: $E_{K_{1}}=2 R^{2} \sigma$, interpolating between the North $A$ and South $\bar{A}$ Poles. Thus, these kinks belong to a different sector in configuration space than the vacua, an evident fact in Cartesian coordinates; see Figures 1-2 [10]:

$$
\Phi^{K_{1}}(x)=\left(0, \frac{1}{\cosh \left[\sigma\left(x-x_{0}\right)\right]}, \pm \tanh \left[\sigma\left(x-x_{0}\right)\right]\right)
$$
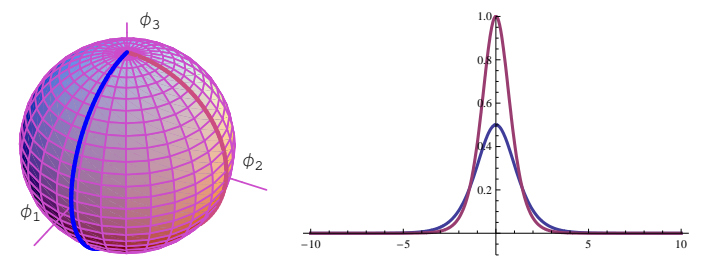

Figure 1: a) $K_{1}$ and $K_{2}\left(\sigma^{2}=\frac{1}{2}\right)$ kink orbits. b) $K_{1}$ (blue) and $K_{2}$ (red) kink energy densities
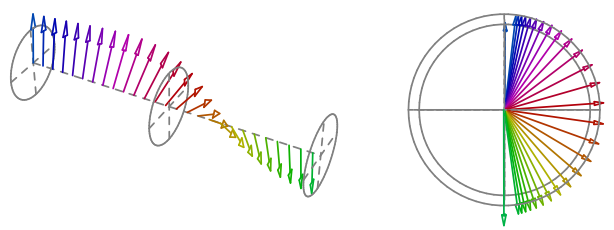

Figure 2: a) $K_{1}$ kinks. b) Perspective from one component of the boundary of the target sphere $\times$ the spatial line infinite cylinder: $\mathbb{S}^{2} \times \mathbb{R}$. The meridian $\varphi_{K_{1}}=\frac{\pi}{2}, \varphi_{K_{1}}=-\frac{\pi}{2}$ is plotted as orthogonal to the spatial line.

The half-meridians $\varphi_{K_{2}}(x)=0$ or $\varphi_{K_{2}}(x)=\pi$ are also good trial orbits in the sense that they provide new kinks of finite energy $E_{K_{2}}=2 R^{2}$

$$
-\frac{d^{2} \theta}{d x^{2}}+\frac{1}{2} \sin 2 \theta=0 \Rightarrow \theta_{K_{2}}(x)=2 \arctan e^{ \pm\left(x-x_{0}\right)}
$$

via finite action solutions of another pendulum equation. These solitary waves live in the same sector of the configuration space as the previous ones and look similar in Cartesian coordinates; see Figures 1-3:

$$
\Phi^{K_{2}}(x)=\left(\frac{1}{\cosh \left(x-x_{0}\right)}, 0, \pm \tanh \left(x-x_{0}\right)\right)
$$

At the $\sigma=1$ limit, all the half meridians $\phi_{K_{\alpha}}(x)=\varphi \in$ $[0,2 \pi)$ are good trial orbits and there is a one-parametric family of solitary waves: $\theta_{K_{\varphi}}(x)=2 \arctan e^{ \pm\left(x-x_{0}\right)}$,

$\Phi^{K_{\varphi}}(x)=\left(\frac{\cos \varphi}{\cosh \left(x-x_{0}\right)}, \frac{\sin \varphi}{\cosh \left(x-x_{0}\right)}, \pm \tanh \left(x-x_{0}\right)\right)$ degenerated in energy: $E_{K_{\varphi}}=2 R^{2}, \forall \varphi$.

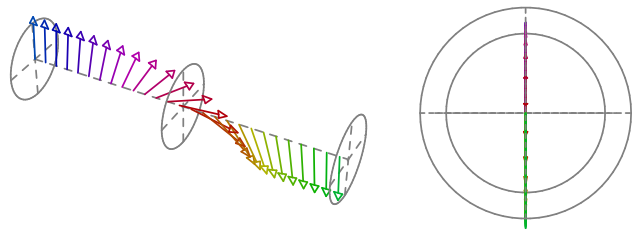

Figure 3: a) $K_{2}$ kinks. b) Perspective from one component of the boundary of the infinite cylinder: $\mathbb{S}^{2} \times \mathbb{R}$. The meridian $\varphi_{K_{2}}=0, \varphi_{K_{2}}=\pi$, is plotted aligned with the spatial line.

\section{THE NON-LINEAR MASSIVE SIGMA MODEL IN ELLIPTIC COORDINATES ON THE SPHERE}

We could now try to search for more kinks even in the case $\sigma^{2}<1$ of distinct masses by using Rajaraman's trial orbit method [3], but instead we shall profit from the fact that the ODE system (6)-(77) is integrable using elliptic coordinates in the $\mathbb{S}^{2}$ sphere. In $\mathbb{S}^{2}$ we fix the two points: $F_{1} \equiv\left(\theta_{f}, \pi\right), F_{2} \equiv\left(\theta_{f}, 0\right), \theta_{f} \in\left[0, \frac{\pi}{2}\right)$. The distance between them is: $d=2 f=2 R \theta_{f}<\pi R$, see Figure 4 .
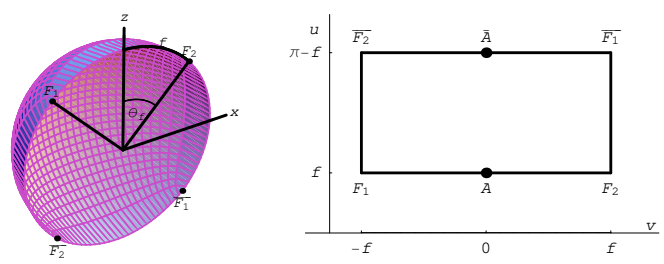

Figure 4: Foci and antipodal foci of the elliptic system of coordinates on the sphere.

Given a point $\Phi \in \mathbb{S}^{2}$, let us consider the distances $r_{1} \in$ $[0, \pi R]$ and $r_{2} \in[0, \pi R]$ from $\Phi$ to $F_{1}$ and $F_{2}$. The elliptic coordinates of $\Phi$ are half the sum and half the difference of $r_{1}$ and $r_{2}: \Phi \equiv\left(u=\frac{r_{1}+r_{2}}{2}, v=\frac{r_{1}-r_{2}}{2}\right)$. The formulae

$$
\begin{aligned}
& \phi_{1}(t, x)=\frac{R}{\mathrm{sf}} \mathrm{susv}, \phi_{2}(t, x)=\frac{R}{\mathrm{cf}} \mathrm{cucv} \\
& \phi_{3}(t, x)= \pm R \sqrt{1-\frac{\mathrm{su}^{2} \mathrm{sv}^{2}}{\mathrm{sf}^{2}}-\frac{\mathrm{cu}^{2} \mathrm{cv}^{2}}{\mathrm{cf}^{2}}}
\end{aligned}
$$

allow one to pass from elliptic to Cartesian coordinates. Here, a simplified notation is used: $\mathrm{su}=\sin \frac{u(t, x)}{R}, \mathrm{cu}=$ $\cos \frac{u(t, x)}{R}, \mathrm{sv}=\sin \frac{v(t, x)}{R}, \mathrm{sf}=\sin \theta_{f}$, etc. The differential arc-length in $\mathbb{S}^{2}$ in this system of coordinates is

$$
d s_{\mathbb{S}^{2}}^{2}=\frac{1}{2} \cdot \frac{\mathrm{su}^{2}-\mathrm{sv}^{2}}{\mathrm{su}^{2}-\mathrm{sf}^{2}} \cdot d u^{2}+\frac{1}{2} \cdot \frac{\mathrm{su}^{2}-\mathrm{sv}^{2}}{\mathrm{sf}^{2}-\mathrm{sv}^{2}} \cdot d v^{2}
$$

Choosing the foci in such a way that $\mathrm{cf}^{2}=\sigma^{2}$, the energy density of our system in elliptic coordinates reads:

$$
\begin{aligned}
\mathcal{E}[u, v]= & \frac{1}{2}\left[\frac{\mathrm{su}^{2}-\mathrm{sv}^{2}}{\mathrm{su}^{2}-\mathrm{sf}^{2}}\left(\frac{d u}{d x}\right)^{2}+\frac{\mathrm{su}^{2}-\mathrm{sv}^{2}}{\mathrm{sf}^{2}-\mathrm{sv}^{2}}\left(\frac{d v}{d x}\right)^{2}\right] \\
& -\frac{f(u)+g(v)}{\mathrm{su}^{2}-\mathrm{sv}^{2}}
\end{aligned}
$$


$f(u(x))=\frac{R^{2}}{2} \mathrm{su}^{2}\left(\mathrm{su}^{2}-\mathrm{sf}^{2}\right), g(v(x))=\frac{R^{2}}{2} \mathrm{sv}^{2}\left(\mathrm{sf}^{2}-\mathrm{sv}^{2}\right)$.

The mechanical analogy demands that we think of $\mathcal{E}$ as the Lagrangian, $x$ as the time, $U[u(x), v(x)]=$ $-V[u(x), v(x)]$ as the mechanical potential energy, and the target manifold $\mathbb{S}^{2}$ as the configuration space. The structure of $\mathcal{E}[u, v]$ is such that we are dealing with a Type I Liouville model [4] on the sphere, i.e., a dynamical system which is Hamilton-Jacobi separable in elliptic coordinates. The kink orbits (finite action trajectories) and the kink profiles ("time" schedules of these trajectories) are given in the Hamilton-Jacobi framework [5]-[ $[6]$ via the quadratures: $\left(p_{u}=\frac{\partial \mathcal{E}}{\partial \dot{u}}, p_{v}=\frac{\partial \mathcal{E}}{\partial \dot{v}}\right)$

$$
\begin{gathered}
\int \frac{\mathrm{sg}\left(p_{u}\right) d u}{\sqrt{2\left(\mathrm{su}^{2}-\mathrm{sf}^{2}\right) f(u)}}-\int \frac{\mathrm{sg}\left(p_{v}\right) d v}{\sqrt{2\left(\mathrm{sf}^{2}-\mathrm{sv}^{2}\right) g(v)}}=R^{2} \gamma_{2} \\
\int \frac{\mathrm{sg}\left(p_{u}\right) \mathrm{su}^{2} d u}{\sqrt{2\left(\mathrm{su}^{2}-\mathrm{sf}^{2}\right) f(u)}}-\int \frac{\mathrm{sg}\left(p_{v}\right) \mathrm{sv}^{2} d v}{\sqrt{2\left(\mathrm{sf}^{2}-\mathrm{sv}^{2}\right) g(v)}}=x+\gamma_{1}
\end{gathered}
$$

\section{NON-TOPOLOGICAL KINKS}

In this way we find a family of non-topological kink (NTK) orbits by integrating the first quadrature (they start and end either at the North or the South Pole, they live in the vacua sectors of the configuration space) parametrized by the integration constant $C=e^{R^{2} \gamma_{2} \mathrm{sf}^{2}}$ :

$C=\left[\frac{\left|\tan \frac{u-f}{2 R} \tan \frac{u+f}{2 R}\right|^{\frac{1}{2 \mathrm{cf}}}}{\left|\tan \frac{u}{2 R}\right|}\right]^{\operatorname{sg} p_{u}}\left[\frac{\left|\tan \frac{v}{2 R}\right|}{\left|\tan \frac{v-f}{2 R} \tan \frac{v+f}{2 R}\right|^{\frac{1}{2 \mathrm{cf}}}}\right]^{\mathrm{s}}$

The kink profiles of these non-topological solitary waves given by the integration of the second quadrature

$$
e^{2\left(x+\gamma_{1}\right) \mathrm{cf}}=\frac{\left|\tan \frac{u-f}{2 R} \tan \frac{u+f}{2 R}\right|^{\operatorname{sg} p_{u}}}{\left|\tan \frac{v-f}{2 R} \tan \frac{v+f}{2 R}\right|^{\operatorname{sg} p_{v}}}
$$

depend on one integration constant, $\gamma_{1}$, which sets the center of each kink, see Figures 5 and 6.
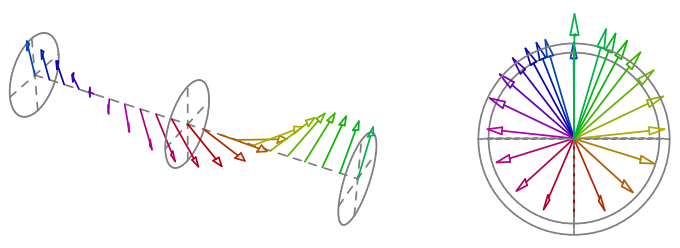

Figure 5: a) A $K_{\gamma_{2}}\left(\left|\gamma_{2}\right|<\infty\right)$ NTK kink. b) Perpendicular cross section of the field variation.

The Hamilton-Jacobi method also provides the energy of the NTK. The Hamilton characteristic function (the
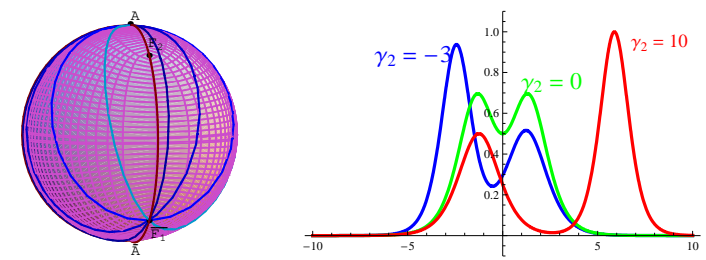

Figure 6: a) Several NTK kink orbits. b) NTK energy densities for three different values of $\gamma_{2}$ : 1) $\gamma_{2}=-3$, highest peak on the left (blue) 2) $\gamma_{2}=0$, symmetrical peaks (green) 3) $\gamma_{2}=10$ highest peak on the right (red).

solution of the Hamilton-Jacobi stationary equation) for zero mechanical energy is:

$$
F(u)+G(v)=(-1)^{\operatorname{sg} p_{u}} R \cos \frac{u}{R}+(-1)^{\operatorname{sg} p_{v}} R \cos \frac{v}{R}
$$

From this function we compute the energy of the NTK kinks:

$$
E_{K\left(\gamma_{2}\right)}=2 R|G(0)-G(f)|+2 R|F(f)-F(\pi-f)|
$$

All the NTK kinks have the same energy and satisfy the kink mass sum rule:

$$
E_{K\left(\gamma_{2}\right)}=2 R^{2}(1+\sigma)=E_{K_{2}}+E_{K_{1}}
$$

${ }^{\operatorname{sg} p_{v}} K_{1}$ and $K_{2}$ are singular kinks that arise at the $\gamma_{2} \rightarrow|\infty|$ limit of the NTK kink moduli space. Their orbits lie on the boundary of the elliptic rectangle and the $v=0$ axis, see Figure 7 . The $K_{1}$ kink orbit is the straight line $v_{K_{1}}(x)=0$. The $K_{2}$ kink orbit, however, is a three-step trajectory: the $u=f, u=\pi-f$, and $v= \pm f$ edges of the rectangle.

Observe that all the NTK orbits starting from the North Pole $A$ (South Pole $\bar{A}$ ) meet at one of the antipodal foci $\bar{F}_{1}-\bar{F}_{2}$ (foci $F_{1}-F_{2}$ ), which are thus conjugate points to the Poles. According to the Morse index theorem, these kinks are unstable, see [7], [8]. The $K_{2}$ orbits also cross the foci and only the kinks of type $K_{1}$ are stable.

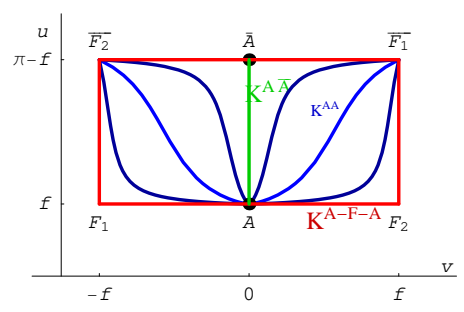

Figure 7: Singular (red and green) and generic (blue) kink orbits in the elliptic rectangle. 


\section{SOLITARY SPIN WAVES}

The Wess-Zumino action

$$
S_{\mathrm{WZ}}=R^{2} \int d t d x \sum_{a=1}^{3} A_{a}[\Phi(t, x)] \frac{\partial \phi_{a}}{\partial t}(t, x)
$$

produces the Euler-Lagrange equation:

$\frac{\partial A_{a}}{\partial t}=\sum_{b=1}^{3}\left(\frac{\delta A_{b}}{\delta \phi_{a}}-\frac{\delta A_{a}}{\delta \phi_{b}}\right) \cdot \frac{\partial \phi_{b}}{\partial t}=\sum_{b=1}^{3} \sum_{c=1}^{3} \varepsilon_{a b c} B_{c}[\Phi] \cdot \frac{\partial \phi_{b}}{\partial t}$

We choose the non-null components of the "vector potential" in the North and South hemispheres of the target space in the form (using $\varepsilon_{\alpha \beta}=-\varepsilon_{\beta \alpha}, \varepsilon_{12}=1$ ):

$$
A_{\alpha}^{ \pm}[\Phi(t, x)]=-\sum_{\beta=1}^{2} \varepsilon_{\alpha \beta} \frac{\phi_{\beta}(t, x)}{2 R\left(\phi_{3}(t, x) \pm R\right)}, \alpha=1,2
$$

A "magnetic monopole field" arises in the target space: $B_{a}[\Phi(t, x)]=\frac{\phi_{a}(t, x)}{R^{3}}$. The combined Euler-Lagrange equations for $S_{\mathrm{WZ}}+S$ are:

$\frac{1}{R} \sum_{b=1}^{3} \sum_{c=1}^{3} \varepsilon_{a b c} \phi_{c}(t, x) \frac{\partial \phi_{b}}{\partial t}(t, x)+\square \phi_{a}(t, x)+\frac{\delta V}{\delta \phi_{a}}(t, x)=0$

At the long wavelength limit $\omega<<\frac{1}{R}$, system (9) becomes the Landau-Lifhsitz equations for ferromagnetism with a dispersion relation: $\omega^{2}(k)=R^{2}\left(k^{2}+1\right)\left(k^{2}+\sigma^{2}\right)$. The connection between the semi-classical (high-spin) limit of the Heisenberg model with the quantum nonlinear sigma model is well established [9]. Thus, our kinks, which are also static finite energy solutions of (9), are solitary spin waves in the low-energy regime of quantum ferromagnets, although the symmetry is contracted from Lorentzian to Galilean.

\section{CONCLUSIONS}

In this letter we have reached the following conclusions about the kink manifold of the 1D massive non-linear $\mathbb{S}^{2}$ sigma model:

1. If the masses of the pseudo-Goldstone particles are equal, there exist a $\mathbb{S}^{1}$-family (fixed the kink center of mass) of topological kinks degenerated in energy living on all the half-meridians of the $\mathbb{S}^{2}$-sphere. When the masses differ, only two pairs of topological kinks survive, each pair of kinks having distinct energy.

2. Even if the masses of the pseudo-Goldstone particles are different, we have shown that there exist a one-parametric family (for fixed CM) of nontopological kinks degenerated in energy by using elliptic coordinates on the $\mathbb{S}^{2}$-sphere.

3. It is also shown that there is a curious kink mass sum rule between the non-topological and topological kinks and that only one of the topological kink pairs is formed by stable kinks.

4. Finally, we have noticed by adding a Wess-Zuminotype term to the action that our kinks are solitary spin waves in the long wavelength limit of ferromagnetic materials.

\section{ACKNOWLEDGEMENTS}

We are grateful to Mariano Santander for enlightening explanations about elliptic coordinates on the sphere and other Cayley-Klein two-dimensional spaces.

We thank also to the Spanish Ministerio de Educacion y Ciencia for partial support under grant FIS2006-09417.
[1] M. Gell-Mann, M. Lèvy, Nuovo Cimento 16 (1960) 705

[2] S. F. Coleman, Comm. Math. Phys. 31 (1973) 259

[3] R. Rajaraman, Phys. Rev. Lett. 42 (1979) 200

[4] A. Perelomov, Integrable Systems of Classical Mechanics and Lie Algebras, Birkhauser, (1992)

[5] H. Ito, Phys. Lett. 112A (1985) 119

[6] A. Alonso Izquierdo, M. A. Gonzalez Leon, J. Mateos Guilarte, J. Phys. A31 (1998) 209
[7] H. Ito, H. Tasaki, Phys. Lett. A113 (1985) 179

[8] J. Mateos Guilarte, Ann. Phys. 188 (1988) 307

[9] F. D. M. Haldane, Phys. Rev. Lett. 50 (1983) 1153

[10] In the web page http://www.usal.es/ mpg/(Mathematica tools) there is a Mathematica file containing several extra figures and formulas concerning this work. 